\title{
ISOTERMAS DE DESSORÇÃO DE SEMENTES DE BETERRABA
}

\author{
Paulo Cesar Corrêa ${ }^{1}$, Gabriel Henrique Horta de Oliveira ${ }^{2}$, Ana Paula Lelis Rodrigues de Oliveira ${ }^{3}$, André Luis \\ Duarte Goneli ${ }^{4}$, Fernando Mendes Botelho ${ }^{5}$
}

\begin{abstract}
RESUMO
Alterações físicas e químicas de produtos agrícolas podem ocorrer durante o armazenamento, levando à perdas quantitativas e qualitativas. Portanto, o conhecimento acerca da relação entre o produto, a temperatura $(\mathrm{T})$ e a umidade relativa (UR) do ambiente é necessário para diminuir possíveis alterações. Dito isto, este trabalho objetivou determinar o modelo matemático mais adequado para predizer o teor de água de equilíbrio $\left(\mathrm{X}_{\mathrm{eq}}\right)$ de sementes de beterraba, $\mathrm{cv}$. "Chata do Egito", em diferentes T e UR. $\mathrm{X}_{\mathrm{eq}}$ foi alcançado por meio do método estático, utilizando soluções salinas saturadas, com valores de UR entre 11 e $96 \%$. Sementes foram colocadas no interior de dessecadores contendo as soluções salinas, posteriormente armazenadas em câmaras BOD em diferentes T $\left(10 ; 20 ; 30 ; 40\right.$ e $\left.50^{\circ} \mathrm{C}\right)$. $\mathrm{X}_{\mathrm{eq}}$ foi obtido quando a variação entre três pesagens consecutivas fosse igual ou menor a $0,01 \mathrm{~g}$. $\mathrm{O}$ teor de água foi determinado pelo método gravimétrico usando estufa a $105 \pm 1{ }^{\circ} \mathrm{C}$ até a massa constante. Cinco modelos matemáticos (Chung Pfost, Copace, Halsey Modificado, Henderson e Henderson Modificado) foram ajustados aos dados experimentais de $\mathrm{X}_{\text {eq }}$. O modelo mais adequado foi escolhido considerando a magnitude do erro médio relativo (MRE), desvio padrão da estimativa (SE) e análise de resíduos. O modelo de Chung-Pfost foi o que mais se ajustou aos dados experimentais, apresentando valores de $8,04 \%$ e $0,62 \%$ b.s. de MRE e SE, respectivamente.
\end{abstract}

Palavras-Chave: Beta vulgaris, equilíbrio higroscópico, modelagem matemática

\section{ABSTRACT}

\section{DESORPTION ISOTHERMS OF SUGAR BEET SEEDS}

Physical and chemical alterations of agricultural products may occur during storage, leading to quantitative and qualitative losses. Thus, knowledge regarding the relationship between the product and temperature (T) and relative humidity ( $\mathrm{RH}$ ) of the environment is required in order to diminish these possible alterations. Being that stated, this work aimed to determine the most adequate mathematical model to represent the equilibrium moisture content $\left(\mathrm{X}_{\mathrm{eq}}\right)$ of sugar beet seed, cv. "Chata do Egito", at different T and RH. $\mathrm{X}_{\mathrm{eq}}$ was achieved by means of static method, using saturated saline solutions, attaining RH values between 11 and $96 \%$. Seeds were placed inside of dissectors containing the saline solutions, afterwards stored at BOD chambers under different $\mathrm{T}\left(10 ; 20 ; 30 ; 40\right.$ and $\left.50^{\circ} \mathrm{C}\right) . \mathrm{X}_{\text {eq }}$ was obtained when variation of three consecutive weightings was equal or lower than $0.01 \mathrm{~g}$. Moisture content was determined by means of gravimetric method using a stove at $105 \pm 1{ }^{\circ} \mathrm{C}$ until constant mass. Five mathematical models (Chung Pfost, Copace, Modified Halsey, Henderson and Modified Henderson) were fitted to experimental data of $\mathrm{X}_{\text {eq }}$. The best model was chosen considering the magnitude of mean relative error (MRE), standard error of the estimate (SE) and residual plots. The Chung-Pfost model was the one that best fitted to experimental data, presenting values of $8.04 \%$ and $0.62 \%$ d.b. for MRE and SE, respectively.

Keywords: Beta vulgaris, hygroscopic equilibrium, mathematical modeling

Recebido para publicação em 07/07/2015. Aprovado em 31/03/2016.

1 - Engenheiro Agrônomo, Prof. Doutor da UFV/Viçosa-MG, copace@ufv.br

2 - Engenheiro Agrícola e Ambiental, Prof. Doutor do IF Sudeste MG/Manhuaçu-MG, gabriel.oliveira@ifsudestemg.edu.br

3 - Bacharel e Licenciada em Química, Prof. Doutora do IF Sudeste MG/Manhuaçu-MG, ana.lelis@ifsudestemg.edu.br

4 - Engenheiro Agrônomo, Prof. Doutor da UFGD/Dourados-MS, andregoneli@ufgd.edu.br

5 - Engenheiro Agrícola e Ambiental, Prof. Doutor da UFMT/Sinop-MT, fernando_eaa@yahoo.com.br 


\section{INTRODUÇÃO}

A lucratividade dos agricultores e agentes atuantes no agronegócio, independentemente da cultura, passa inicialmente pela viabilidade e qualidade das sementes a serem utilizadas. Assim, além da escolha dos precursores das melhores sementes, o correto armazenamento das mesmas é imprescindível, de modo a permitir a utilização das sementes em momento oportuno.

A armazenagem segura de sementes requer o acompanhamento da temperatura e da umidade relativa do armazém, uma vez que a combinação destes dois parâmetros rege a sorção de água nas sementes. Por meio da sorção, o teor de água pode incrementar ou decrescer durante o armazenamento, resultando em maior ou menor atividade de insetos e microrganismos, alterando a capacidade germinativa da semente (Lopes \& Macedo 2008). Essas alterações ocorrem em razão da característica higroscópica das sementes, ou seja, que possuem a capacidade de dessorver ou adsorver água.

Os modelos matemáticos são imprescindíveis pois relatam o teor de água do produto em determinada condição ambiental sem a necessidade de realizar testes onerosos e demorados. Além disso, em conjunto com a previsão do tempo, pode-se antever em um espaço de tempo longo o que poderá ocorrer com as sementes armazenadas, sendo informação importante para a tomada de decisão do profissional responsável de sementes.

Os modelos matemáticos possibilitam a construção das isotermas de sorção. Essas são ferramentas valiosas para predizer quais reações acarretarão no decréscimo da estabilidade do material em um dado teor de água, possibilita a seleção de ingredientes para alterar a atividade de água, para aumentar a estabilidade do alimento e podem ser usadas para determinar o ganho ou a perda de umidade em uma embalagem de conhecida permeabilidade à umidade (Bell \& Labuza 2000).

A cultura da beterraba utiliza grande quantidade de sementes em sua implantação, em que boa parte de sua aquisição se dá atualmente por importação, onerando os produtores e consumidores. Em razão da inexistência de trabalhos acerca da higroscopicidade de sementes de beterraba e da necessidade de se armazenar estas de forma adequada, objetivou-se com este trabalho determinar o melhor modelo matemático para predizer o teor de água de sementes de beterraba em diferentes condições ambientais, de modo a se obter as isotermas de dessorção.

\section{MATERIAL E MÉTODOS}

O presente trabalho foi desenvolvido no Laboratório de Propriedades Físicas e Qualidade de Produtos Agrícolas pertencente ao Centro Nacional de Treinamento em Armazenagem (CENTREINAR), localizado na Universidade Federal de Viçosa, Viçosa, MG.

Com vista à obtenção do teor de água de equilíbrio das sementes de beterraba pelo processo de dessorção, foi utilizado o método estático gravimétrico (Brasil, 2009). Foram utilizadas diferentes condições de temperatura $(10 ; 20 ; 30$; 40 e $50 \pm 1{ }^{\circ} \mathrm{C}$ ) e umidade relativa (entre 11 e 96 $\%$ ) até que o produto atingisse o teor de água de equilíbrio com a condição do ar especificada. As umidades relativas (ou atividade de água, quando no equilíbrio) descritas foram fornecidas por soluções salinas saturadas (Quadro 1).

Cada amostra consistiu de $20 \mathrm{~g}$ de sementes (cv. Chata do Egito), sendo realizado em triplicata. Durante a dessorção, as amostras foram pesadas periodicamente e o equilíbrio higroscópico foi alcançado quando a variação de massa em três pesagens consecutivas foi menor ou igual a 0,01 g. O teor de água do produto foi então determinado pelo método gravimétrico utilizando-se uma estufa com circulação forçada de ar a $105 \pm 1{ }^{\circ} \mathrm{C}$ por 24 $\mathrm{h}$ em três amostras de $50 \mathrm{~g}$, de acordo com Brasil (2009), com modificações.

Aos dados experimentais do teor de água de equilíbrio das sementes de beterraba obtido para cada condição psicrométrica do ar foram ajustados os modelos matemáticos apresentados no Quadro 2.

Para o ajuste dos modelos matemáticos, foi realizada análise de regressão não-linear pelo método Gauss Newton. Para verificar o grau de ajuste dos modelos, foram consideradas as magnitudes do desvio padrão da estimativa (SE) e do erro médio relativo (MRE) sendo que, de modo geral, quanto menores forem as magnitudes desses índices melhor o ajuste do modelo aos dados observados. Os valores de MRE e SE para cada modelo foram calculados pelas Equações 6 e 7, respectivamente: 
Quadro 1. Sais utilizados para a manutenção das umidades relativas estabelecida nos dessecadores para a determinação do equilíbrio higroscópico de sementes de beterraba pelo método estático

\begin{tabular}{cccccc}
\hline \multirow{2}{*}{ Solução salina } & \multicolumn{5}{c}{ Temperatura $\left({ }^{\circ} \mathrm{C}\right)$} \\
\cline { 2 - 6 } & 10 & 20 & 30 & 40 & 50 \\
\hline $\mathrm{LiCl}$ & 0,13 & -- & 0,11 & 0,12 & 0,11 \\
$\mathrm{CaCl}_{2}$ & 0,40 & 0,35 & -- & -- & -- \\
$\mathrm{Ca}\left(\mathrm{NO}_{3}\right)_{2}$ & 0,59 & 0,55 & -- & -- & -- \\
$\mathrm{NH}_{4} \mathrm{Cl}$ & 0,81 & -- & -- & -- & -- \\
$\mathrm{NaCl}$ & -- & 0,76 & 0,76 & 0,75 & 0,75 \\
$\mathrm{KBr}$ & -- & 0,84 & -- & -- & -- \\
$\mathrm{K}_{2} \mathrm{SO}_{4}$ & -- & -- & -- & 0,96 & -- \\
$\mathrm{MgCl}_{2}$ & -- & -- & 0,32 & -- & -- \\
$\mathrm{KNO}_{2}$ & -- & -- & 0,47 & -- & -- \\
$\mathrm{KNO}_{3}$ & 0,96 & 0,93 & 0,91 & -- & -- \\
$\mathrm{MgCl}_{2} \times 6 \mathrm{H}_{2} \mathrm{O}$ & -- & -- & -- & 0,32 & 0,31 \\
$\mathrm{Na}_{2} \mathrm{Cr}_{2} \mathrm{O}_{7}$ & -- & -- & -- & 0,50 & 0,46 \\
\hline
\end{tabular}

Fonte: Greenspan (1977)

Quadro 2. Modelos matemáticos para representação das curvas de equilíbrio higroscópico

\begin{tabular}{lll}
\hline Nome do modelo (Fonte) & Equação & $N^{\mathrm{o}}$ \\
\hline $\begin{array}{l}\text { Chung-Pfost } \\
\text { (Pfost et al., 1976) }\end{array}$ & $X_{e q}=a-b \ln [-(T+c) \ln U R]$ \\
$\begin{array}{l}\text { Copace } \\
\text { (Corrêa et al., 1995) }\end{array}$ & $X_{\theta q}=\exp (a-b T+c U R)$ \\
$\begin{array}{l}\text { Halsey Modificado } \\
\text { (Iglesias \& Chirife, 1976) }\end{array}$ & $X_{\theta q}=[\exp (a-b T /-\ln U R)]^{1 / c}$ \\
$\begin{array}{l}\text { Henderson } \\
\text { (Henderson, 1952) } \\
\text { Henderson Modificado } \\
\text { (Thompson et al., 1968) }\end{array}$ & $X_{e q}=[\ln (1-U R) /-a(T+273,16)]^{1 / c}$ \\
\hline
\end{tabular}

em que,

$\mathrm{X}_{\mathrm{eq}}=$ teor de água de equilíbrio, \% b.s.;

$\mathrm{UR}=$ umidade relativa, decimal;

$\mathrm{T}=$ temperatura; $\mathrm{e}$

${ }^{\circ} \mathrm{C} ; a, b, c=$ coeficientes de ajuste dos modelos que dependem do produto.

$M R E=\frac{100}{n} \sum_{i=1}^{n}\left(\frac{|Y-\hat{Y}|}{Y}\right)$

$S E=\sqrt{\frac{\sum_{i=1}^{n}(Y-\hat{Y})^{2}}{G L R}}$ em que,

$\mathrm{Y}=$ valor observado experimentalmente;

$\hat{Y}=$ valor estimado pelo modelo;

$\mathrm{n}=$ número de dados observados; e

GLR $=$ graus de liberdade do resíduo (número de dados observados menos o número de parâmetros do modelo). 
Além do erro médio relativo e do desvio padrão da estimativa, foram considerados ainda a magnitude do coeficiente de determinação $\left(\mathrm{R}^{2}\right)$ e o comportamento da distribuição dos resíduos (aleatório ou tendencioso).

\section{RESULTADOS E DISCUSSÃO}

De modo a se determinar o melhor modelo matemático durante a modelagem, busca-se o que apresenta o melhor ajuste possível aos dados experimentais, tendo-se em mente os valores do erro de sua estimativa e a distribuição dos resíduos. Para a averiguação do erro associado à estimativa do modelo, a literatura especializada relata que, para se obter um ajuste satisfatório, o valor de MRE deve ser menor que 10\% (Henao et al., 2009; Rosa et al., 2010; Costa et al., 2015). Dito isso, nota-se que apenas o modelo de Chung-Pfost é satisfatório (Quadro 3).

De acordo com Draper \& Smith (1998), a habilidade de um modelo para descrever adequadamente um processo físico é inversamente proporcional aos valores de SE. O modelo de Chung-Pfost foi o que obteve o menor valor de SE, sendo apto a descrever a dessorção de sementes de beterraba.

Por fim, de modo a selecionar adequadamente o modelo para representar o fenômeno estudado, deve-se levar em consideração a distribuição dos resíduos. Essas são as diferenças entre os valores observados experimentalmente e os estimados pelo modelo, representados como uma função dos níveis estimados. Essa análise possibilita avaliar como o modelo estima os valores da variável em estudo. Um modelo é considerado aceitável se os valores residuais se encontram em uma zona horizontal perto de zero formando distribuições aleatórias (Corrêa et al., 2014). Estes mesmos autores relatam que, se as distribuições dos resíduos formam figuras geométricas, apresentam regiões em que o modelo subestima ou superestima a condição real ou, se tendem a se acumular em um ponto fora do eixo, a distribuição dos seus resíduos é considerada tendenciosa e o modelo inadequado para representar o fenômeno em questão.

Nota-se que na distribuição dos resíduos do modelo Chung-Pfost não é possível perceber nenhuma tendência em seus resíduos, sendo distribuído aleatoriamente na faixa de teor de água de equilíbrio, com resíduos de valores positivos e negativos (Figura 1).

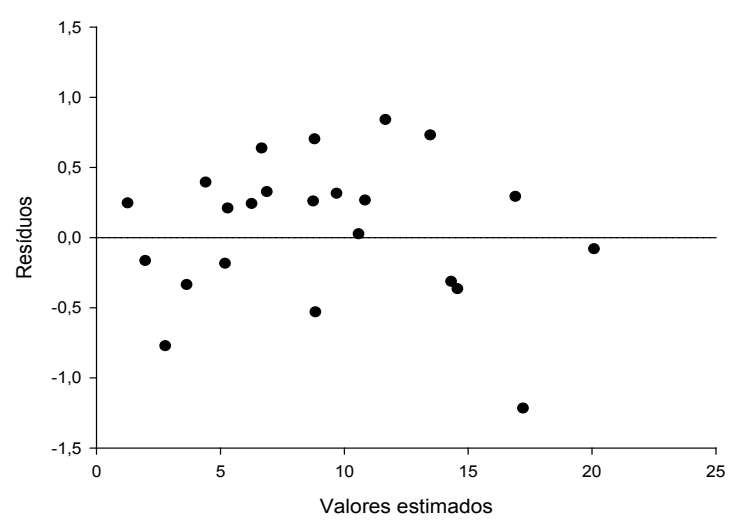

Figura 1. Comportamento da distribuição dos resíduos do modelo Chung-Pfost avaliados para obtenção das isotermas de dessorção das sementes de beterraba.

O modelo de Chung-Pfost também representou, de forma satisfatória, a higroscopicidade de sementes de quiabo (Goneli et al., 2010), de

Quadro 3. Coeficientes de ajuste e parâmetros estatísticos dos modelos de equilíbrio higroscópico para as sementes de beterraba, obtidos por dessorção

\begin{tabular}{ccccccc}
\hline Modelo & & Parâmetros & MRE & SE & R \\
& $\mathrm{a}$ & $\mathrm{b}$ & $\mathrm{c}$ & $(\%)$ & $\begin{array}{c}\mathrm{R}^{2} \\
(\%)\end{array}$ \\
\hline Chung-Pfost & 19,6937 & 3,7919 & 9,6156 & 8,04 & 0,62 & 98,67 \\
Copace & 1,3901 & 0,0113 & 1,8036 & 15,90 & 0,92 & 97,08 \\
Halsey Modificado & 6,5902 & 0,0364 & 2,9972 & 28,26 & 1,47 & 92,49 \\
Henderson & 0,0001 & 1,7191 & - & 19,63 & 1,40 & 92,96 \\
Henderson Modificado & 0,0003 & 25,2966 & 1,8536 & 11,91 & 0,70 & 98,31 \\
\hline
\end{tabular}


sementes de caju-de-árvore-de-cerrado (Caetano et al., 2012), para sementes de trigo (Li, 2012) e para sementes de algodão (Oliveira et al., 2013).

Verifica-se a influência da temperatura sobre a higroscopicidade das sementes de beterraba em que, para se atingir um mesmo teor de água de equilíbrio, o aumento da temperatura requer o aumento da umidade relativa. De forma análoga, para um valor constante de umidade relativa, o teor de água de equilíbrio diminui com o aumento da temperatura (Figura 2).

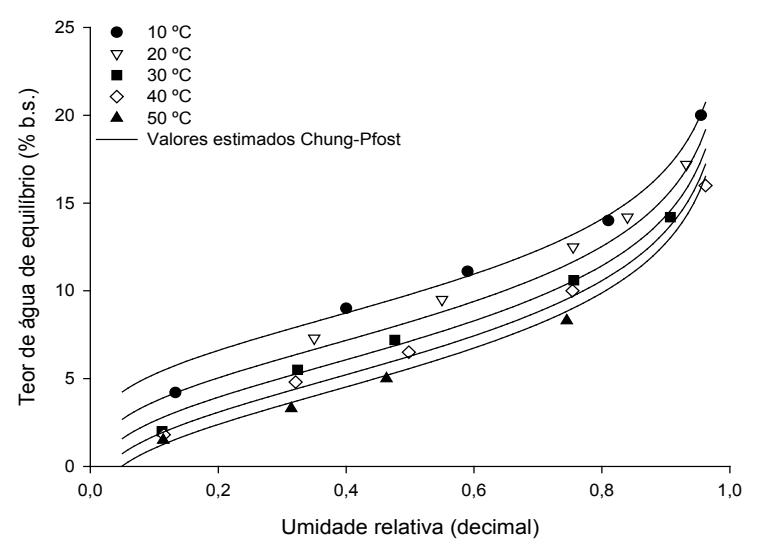

Figura 2. Valores observados e estimados pelo modelo de Chung-Pfost, do teor de água de equilíbrio de sementes de beterraba, obtidos por dessorção.

Segundo Mohsenin (1986), ao passo que ocorre um incremento na temperatura, a vibração das moléculas sofre um aumento, elevando a distância entre estas, diminuindo assim a atração entre as moléculas, fazendo com que a quantidade de água sorvida mude à medida que ocorre variação na temperatura a uma dada umidade relativa. Em trabalho posterior, Palipane \& Driscoll (1992) indicaram que o aumento da temperatura acarreta em maiores níveis energéticos das moléculas de água, tornando-as termodinamicamente menos estáveis, facilitando a quebra da ligação entre a água e os locais de sorção, culminando na diminuição do teor de água do produto.

Verifica-se que houve boa correspondência entre os dados estimados pelo modelo de Chung-Pfost e os observados experimentalmente. Ressalta-se que o modelo de Chung-Pfost não permite a predição dos teores de água quando a umidade relativa tende a zero, sendo uma limitação do modelo para o caso de dessorção de sementes de beterraba (Figura 2).

As isotermas de dessorção de sementes de beterraba podem ser classificadas como do tipo II (Figura 3). De acordo com Labuza \& Altunakar (2007), a forma das isotermas do tipo II é causada por efeitos sinérgicos da lei de Raoult, efeitos capilares e interações de umidade na superfície do material. Estes autores relatam ainda que este tipo de isoterma descreve duas regiões, uma entre 0,2 e 0,4 de umidade relativa no equilíbrio e outra entre 0,6 e 0,7 . Estas regiões são resultado de efeitos físico-químicos, como por exemplo, a criação de multicamadas e o preenchimento de poros pequenos (região 0,2-0,4), seguido de enchimento dos poros maiores e dissolução de solutos (região 0,6-0,7) (Labuza \& Altunakar 2007).

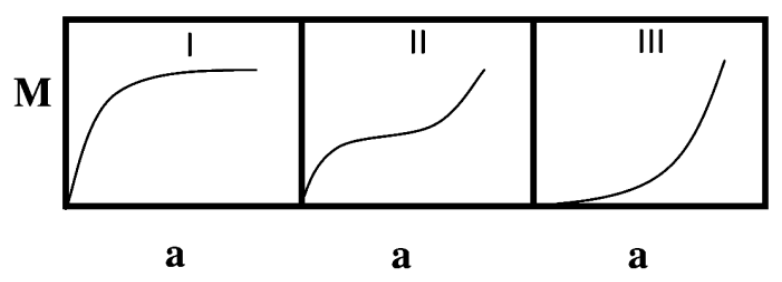

Figura 3. Três tipos de isotermas de sorção demonstrando o teor de água adsorvente (M) versus a atividade de água (a). Fonte: Labuza \& AltunaZkar (2007).

\section{CONCLUSÕES}

- O teor de água de equilíbrio das sementes de beterraba aumenta com a umidade relativa e a temperatura.

- O modelo de Chung-Pfost Pfost foi o que mais se ajustou aos dados experimentais, apresentando valores de $8,04 \%$ e $0,62 \%$ b.s. de MRE e SE, respectivamente.

- As isotermas de dessorção de sementes de beterraba são classificadas como do tipo II.

\section{AGRADECIMENTOS}

Os autores agradecem ao CNPq - Conselho Nacional de Desenvolvimento Científico e Tecnológico e à FAPEMIG - Fundação de Amparo 
à Pesquisa do Estado de Minas Gerais, pelo apoio financeiro.

\section{REFERÊNCIAS BIBLIOGRÁFICAS}

BELL, L.N.; LABUZA, T.P. Moisture Sorption: Practical Aspects of Isotherms Measurements and Use. St. Paul, MN: AACC International Publishing, 2000. 123p.

BRASIL, Ministério da Agricultura e Reforma Agrária. Secretaria Nacional de Defesa Agropecuária. Regras para análises de sementes. Brasília: MAPA, 2009. 399p.

BRUNAUER, S. The Adsorption of Gases and Vapors. Princeton, NJ: Princeton University Press, 1945. 528p.

CAETANO, G.S.; SOUSA, K.A.; RESENDE, O.; SALES, J.F.; COSTA, L.M. Higroscopicidade de sementes de caju-de-árvore-do-cerrado. Pesquisa Agropecuária Tropical, Goiânia, v.42, n.4, p.437445. 2012.

CORRÊA, P.C.; BOTELHO, F.M.; BOTELHO, S.C.C.; GONELI, A.L.D. Isotermas de sorção de água de frutos de Coffea canephora. Revista Brasileira de Engenharia Agrícola e Ambiental, Campina Grande, v.18, n.10, p.1047-1052. 2014.

CORREAA, P.C.; MARTINS, D.S.R.; MELO, E.C. Umigrãos: Programa para o cálculo do teor de umidade de equilíbrio para os principais produtos agrícolas. Viçosa, Brazil: Universidade Federal de Viçosa, Centreinar. 1995.

COSTA, J.M.G.; SILVA, E.K.; HIJO, A.A.C.T; AZEVEDO, V.M.; BORGES, S.V. Physical and Thermal Stability of Spray-Dried Swiss Cheese Bioaroma Powder. Drying Technology, v.33, p.346-354, 2015.

DRAPER, N.R.; SMITH, H. Applied regression analysis. New York: John Wiley \& Sons, 1998. $736 \mathrm{p}$.

GONELI, A.L.D.; CORRÊA, P.C.; OLIVEIRA,
G.H.H.; BOTELHO, F.M. Water desorption and thermodynamic properties of okra seeds. Transactions of the ASABE, St. Joseph, v.53, n.1, p.191-197. 2010.

GREENSPAN, L. Humidity fixed points of binary satured aqueous solutions. Journal of Research of the National Bureau of Standards, v.81, n.1, p.8996, 1977.

HENAO, J.D.; QUEIROZ, M.R.; HAJ-ISA, N.M. A. Umidade de equilíbrio de café cereja descascado baseada em métodos estático e dinâmico. Revista Brasileira de Engenharia Agrícola e Ambiental, Campina Grande, v.13, p.470-476. 2009.

HENDERSON, S.M.A basic concept of equilibrium moisture. Agricultural Engineering, v.33, n.1, p.2933, 1952.

IGLESIAS, H.; CHIRIFE, J. Prediction of the effect of temperature on water sorption isotherms of food material. Journal of Food Technology, Malden, v.11, n.2, p.109-116. 1976.

LABUZA, T.P.; ALTUNAKAR, B. Water Activity Prediction and Moisture Sorption Isotherms. In: BARBOSA-CÁNOVAS, G.V.; FONTANA JR., A.J.; SCHMIDT, S.J.; LABUZA, T.P. (eds.). Water Activity in Foods: Fundamentals and Applications. Ames: Blackwell Publishing Professional, 2007, p.109-154.

LOPES, J.C.; MACEDO, C.M.P. Germinação de sementes de couve chinesa sob influência do teor de água, substrato e estresse salino. Revista Brasileira de Sementes, Londrina, v.30, n.3, p.79-85. 2008.

LI, X. The hygroscopic properties and sorption isosteric heats of different chinese wheat types. Journal of Food Research, Toronto, v.1, n.2, p.8298. 2012.

MOHSENIN, N.N. Physical properties of plant and animal materials. New York: Gordon and Breach Publishers, 1986. 841p.

OLIVEIRA， D.E.C.; RESENDE， O.; 
SMANIOTTO, T.A.S.; CAMPOS, R.C. Isotermas e calor isostérico das sementes de algodão com línter e sem línter. Revista Brasileira de Produtos Agroindustriais, Campina Grande, v.15, n.3, p.283292. 2013.

PFOST, H.B.; MOURER, S.G.; CHUNG, D.S.; MILLIKEN, G.A. Summarizing and reporting equilibrium moisture data for grains. ASAE Paper No. 76-3520. St Joseph, Mich.: ASAE, 1976.

PALIPANE, K.B.; DRISCOLL, R.H. Moisture sorption characteristics of in shell macadamia nuts.
Journal of Food Engineering, California, v.18, p.63-76. 1992.

ROSA, G.S.; MORAES, M.A.; PINTO, L.A.A. Moisture sorption properties of chitosan. Lebensmittel-Wissenschaft und - Technologie, Suiça, v.43, p.415-420. 2010.

THOMPSON, T.L.; PEART, R.M.; FOSTER, G.H. Mathematical simulation of corn drying: A new model. Transactions of the ASAE, St. Joseph, v.11, n.4, p.582-586. 1968. 